\title{
N-Acetylaspartate Metabolism Underlays the Structural and Functional Units of the Vertebrate Brain: A Bioenergetic Rationale for Clinical Observations of Changes in the Neuronal Biomarker "NAA" in many Human Brain Disorders
}

\section{Morris H. Baslow ${ }^{1 *}$ and Alessandro P. Burlina ${ }^{2}$}

${ }^{1}$ Nathan S. Kline Institute for Psychiatric Research, Center for neurochemistry, 140 Old Orangeburg Road, Orangeburg, New York 10962, USA

${ }^{2}$ Consultant in Neurometabolic Hereditary Diseases at the University Hospital of Padua, Neurological Unit, St. Bassiano Hospital, Via dei Lotti 40, I-36061 Bassano del Grappa, Italy

\begin{abstract}
The "structural unit" of the vertebrate brain has been identified as a multi-cellular unit, formed from a single neuron and at least one each of its associated macroglial chaperone and vascular endothelial cells, which exhibits most of the fundamental properties of a brain. These properties include its genetic codes, cellular associations, mechanisms of neuronal information encoding, and its "operating system", a homeostatic energy supply mechanism that enables neurons to continuously communicate with other neurons regardless of the bioenergetic demands made on the neural network in any part of the brain at any time. A structural unit is defined by three cell types required for the unique tri-cellular metabolism of $\mathrm{N}$-acetylaspartate (NAA) and $\mathrm{N}$-acetylaspartylglutamate (NAAG) including neurons, oligodendrocytes and astrocytes, and by their physiological roles operating in a four-cell domain which also includes vascular endothelial cells. A "functional unit" of the brain is a two-neuron entity, defined by the minimum number of neurons required for rapid intercellular communication.

Thus, each functional unit is formed by the process of synaptogenesis from two single-neuron structural units and represents the smallest unit that exhibits all of the basic signaling properties present in a complex brain. These properties include all mechanisms of neuronal connectivity, information storage, and signaling. Since a structural unit is defined by NAA and NAAG intercellular metabolism and physiology, and two structural units form a functional unit, the NAA-NAAG system is intimately associated with all normal brain activities as well as all brain disorders. In this review, the hierarchal structural and functional units of the brain are described. In addition, a bioenergetic rationale for using the NAA-NAAG metabolic system as a biomarker for neuronal abundance and/or viability is presented, and examples of some human diseases that can be traced to interference with one or more components of structural or functional units are provided.
\end{abstract}

Keywords: Brain; Canavan disease; Functional units; Hypoacetylaspartia; NAA; NAAG; Neurons; Structural units; Brain disorders

Abbreviations: Ac: Acetate; AcCoA: Acetyl coenzyme A; AD: Alzheimer's Disease; ALS: Amyotrophic Lateral Sclerosis; Asp: Aspartate; AQP4: Aquaporin 4; ASPA: Aspartoacylase; BBB: Blood Brain Barrier; CD: Canavan Disease; CNS: Central Nervous System; CSF: Cerebro Spinal Fluid; DSD: Dendritic-Synaptic-Dendritic; ECF: Extra Cellular Fluid; fMRI: Functional Magnetic Resonance Imaging; Gj: Gap Junction; Glc: Glucose; Glu: Glutamate; GRM3: Metabotropic Glu Receptor 3; MRI: Magnetic Resonance Imaging; MRS: Magnetic Resonance Spectroscopy; MRSI: Magnetic Resonance Spectroscopic Imaging; NAA: N-Acetylaspartate; NAAG: N-Acetylaspartylglutamate; Syn: Synapse; T: Tesla; Tj: Tight Junction; tNAA: Total NAA and NAAG

\section{Introduction}

The brain is a complex organ whose function is the processing of information and initiation of appropriate behaviors. To achieve this complexity, a brain is formed from much less complex hierarchal structural subunits, each containing a single neuron, and with structural units connected to one another in a variety of ways. The "structural unit" of the vertebrate brain has been identified on the basis of the unique tri-cellular metabolism of two neuronal metabolites [1]. One, an acetylated amino acid, $\mathrm{N}$-acetylaspartate (NAA) that is synthesized from L-aspartate (Asp) and acetyl coenzyme A (AcCoA) [2], and the other is a ligase generated L-glutamate (Glu) adduct of NAA and Glu, $\mathrm{N}$-acetylaspartylglutamate (NAAG) $[3,4]$.
While these substances are synthesized almost exclusively by neurons, neurons generally cannot hydrolyze them, and they are released to extra-cellular fluid (ECF) for their catabolism [5]. In brain, released NAA is targeted to oligodendrocytes [6] where it is hydrolyzed by oligodendrocyte aspartoacylase (ASPA) [7] producing Asp and acetate (Ac). NAAG is targeted to the metabotropic Glu receptor 3 (GRM3, mGluR3), a receptor highly expressed in astrocytes, and is then hydrolyzed by astrocyte NAAG peptidase [8,9], forming NAA and Glu, with most of the NAA released to ECF and subsequently hydrolyzed by oligodendrocyte ASPA. As a result, this unique metabolic sequence in brain requires three cell types for its completion, neurons, astrocytes, and oligodendrocytes, cell types which also constitute most of the cellular volume of the central nervous system (CNS).

${ }^{*}$ Corresponding author: Morris $\mathrm{H}$. Baslow, PhD, Nathan S. Kline Institute for Psychiatric Research, Center for Neurochemistry, 140 Old Orangeburg Road, Orangeburg, New York 10962, USA, Tel: 845-398-5471; Fax: 845-398-5472; E-mail: Baslow@nki.rfmh.org

Received December 05, 2011; Accepted January 09, 2011; Published January 14, 2011

Citation: Baslow MH, Burlina AP (2012) N-Acetylaspartate Metabolism Underlays the Structural and Functional Units of the Vertebrate Brain: A Bioenergetic Rationale for Clinical Observations of Changes in the Neuronal Biomarker "NAA" in many Human Brain Disorders. Bioenerg Open Access 1:102. doi:10.4172/2167. 7662.1000102

Copyright: (c) 2012 Baslow MH, et al. This is an open-access article distributed under the terms of the Creative Commons Attribution License, which permits unrestricted use, distribution, and reproduction in any medium, provided the original author and source are credited. 
Citation: Baslow MH, Burlina AP (2012) N-Acetylaspartate Metabolism Underlays the Structural and Functional Units of the Vertebrate Brain: A Bioenergetic Rationale for Clinical Observations of Changes in the Neuronal Biomarker "NAA" in many Human Brain Disorders. Bioenerg

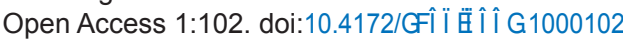

Many investigators use NAA and NAAG as biomarkers of neuronal abundance and/or integrity and have described specific positive or negative changes in NAA and/or NAAG associated with both performance-enhanced normal brain functioning [10], as well as with abnormal brain functioning [11]. We believe that a better understanding of the physiological significance of the NAA metabolic system will improve our ability to make practical sense of such findings, and perhaps lead to targeted remedies for certain pathologies. Clearly, in this paper we cannot include all the evidence with regard to NAA and NAAG metabolism accumulated over the past six decades since their discovery. The results of early studies of these substances have been reviewed [12-14]. In the following sections, we attempt to integrate highlights of what is known about the metabolic and physiological functions of the tri-cellular NAA metabolic system with various experimental and clinical findings related to specific components of this system.

\section{Discussion}

\section{NAA metabolism and brain function}

In this section, we describe evidence that led to the concept that the NAA metabolic system is the "operating system" of the brain [15] and that this system not only provides for the bioenergetic requirements of activated neurons, but also defines and underlies the basic hierarchal structural and functional subunits of the brain.

The NAA-NAAG metabolic-physiological system defines the "structural unit" of the brain: NAA and NAAG are important intracellular osmolytes in neurons in that they are present in very high concentrations, about 20 and $1 \mathrm{mM}$ respectively, are released at about $0.1 \%$ of their $\mathrm{mM}$ content $/ \mathrm{min}$ and as a result turn over approximately every 14-16 h. At this rate of release coupled with their rapid hydrolysis, $>99.9 \%$ of these substances is present within the neuronal cytosol at any given moment [14]. Each molecule of NAA and NAAG released to ECF is associated with a hydration shell of 32 and 53 molecules of obligated water respectively [16]. In addition, NAA and NAAG are anionic substances and therefore also associated with a cation at physiological $\mathrm{pH}$, probably metallic, along with additional cation-obligated water molecules [17]. For example, each NAA molecule released by neurons can be described as the complex; "NAA- $32 \mathrm{H}_{2} \mathrm{O}-\mathrm{Me}^{+} 5 \mathrm{H}_{2} \mathrm{O}$ ", and each NAAG as the complex; "NAAG- $53 \mathrm{H}_{2} \mathrm{O}-\mathrm{Me}^{+} 5 \mathrm{H}_{2} \mathrm{O}$ ". Indeed, all hydrophilic solutes in living systems exist as such water complexes, and therefore each complex can also be considered as being a different "species" of water, each exhibiting its own unique set of dimensional and diffusion properties [18].

Stimulated neurons release both NAA and NAAG down their gradients into ECF at about a 10:1 ratio, along with their obligated water molecules. Therefore, it has been proposed that a primary physiological function of released NAA, and a secondary function of NAAG, based on their co-transport of water molecules, is that of a molecular water pump (MWP) mechanism for transport of neuronal metabolic water into ECF against a water gradient $[15,19,20]$. This mechanism is needed since neurons are especially insulated from the movement of free water across their plasma membranes in that they do not express aquaporin-4 (AQP4) free water channels [21], nor any of the other 12 known AQPs in any abundance [22], and experimentally appear to be largely water impermeable [23]. Although a MWP role proposed for NAA and NAAG remains controversial, there is currently no other hypothesis that explains what is known about their coupled synthesis and complex inter-compartmental cycling. Whereas NAA in brain was first identified in 1956, at this time, some six decades later, its biological function is still considered to be "not fully understood" [11,24].
Based on the MWP hypothesis, it follows that when neuronal metabolic and other water is transported to ECF in the form of NAA or NAAG bound water that the water must then be released for transport into the vascular water sink, and the constituent MWP amino acids recycled into NAA and NAAG in order to maintain the pump mechanism. Evidence of such recycling exists.

Released NAA-water is targeted to oligodendrocytes where it is hydrolyzed by ASPA. NAA-water itself diffuses relatively slowly having an apparent diffusion coefficient (ADC) of $0.25\left(\mathrm{x} 10^{-3} \mathrm{~mm}^{2} / \mathrm{s}\right)$ [16], and is too large to pass through AQP4 channels present on vascular endothelial cell surfaces. However, free water released by the action of ASPA has a diffusion coefficient (D) of 3.64 at $37^{\circ} \mathrm{C}$ [25], and thus not only diffuses 14.6 times faster than NAA-water, but can also pass down a water gradient via AQP4 channels into the vascular water sink for its rapid removal from brain. As part of this water-release and transport process, the liberated Ac is taken up by oligodendrocytes and further metabolized, and the Asp is taken up by neurons and recycled into NAA and NAAG [14].

Released NAAG-water has a high affinity for the GRM3 receptor on astrocyte and neuronal surfaces and therefore it has been proposed that the primary physiological function of exported NAAG is to activate astrocytes via this Glu receptor. However, the GRM3-docked NAAG itself appears to be inactive, and astrocyte activation is postulated to occur only after enzymatic liberation of its Glu moiety by NAAG peptidase [26-28], an enzyme expressed in astrocytes and that may be complexed with the GRM3 surface receptor. Thus, the activation of astrocytes by neuron-released NAAG may be indirect, being initiated by the action of the enzyme liberated Glu which then results in the release of second astrocytic messengers to the vascular endothelial cells forming the capillaries, inducing focal hyperemic responses at the level of capillaries [29-33]. Any released Glu can be taken up by astrocytes, converted into glutamine and then recycled to neurons in this form. Most of the NAA-water is released to ECF and is then hydrolyzed by oligodendrocyte ASPA for water release and recycling of Asp.

Since stimulated neurons release both NAAG and NAA to ECF, the outcome of these metabolic-physiological sequences is to provide individual neurons with mechanisms for (A) exporting metabolic water, (B) increasing focal sink capacity for its metabolic waste products as well as heat, and (C) obtaining sufficient energy supplies from the vascular system regardless of their momentary state of activation. This ability to rapidly increase focal supplies of Glc and $\mathrm{O}_{2}$ is of utmost importance since axons have less than $1 \mathrm{~min}$, and neuron cell bodies only a few min supply of available energy at any given moment [34]. Without this mechanism the ability of neurons to communicate would be rapidly degraded and cognitive functioning negatively affected. The changes in focal blood flow upon acute stimulation of specific sets of neurons can be visualized using functional magnetic resonance imaging (fMRI). Based on use of this investigative tool, stimulationinduced hyperemic responses that increase the ratio of oxyhemoglobin to deoxyhemoglobin result in an increase in the focal MR water signal, forming the basis of the blood oxygenation-level dependent (BOLD) effect widely used in studies of brain activation [31]. The component cells of a structural unit involved in this bioenergetic process, neurons, astrocytes and vascular endothelial cells, along with microglial cells have also been described as the "neurovascular unit" of the brain [30].

The NAA-NAAG homeostatic metabolic-physiological system has been proposed to be the operating system in brain in that it is required to supply energy as needed and to remove metabolic wastes in order 
Citation: Baslow MH, Burlina AP (2012) N-Acetylaspartate Metabolism Underlays the Structural and Functional Units of the Vertebrate Brain: A Bioenergetic Rationale for Clinical Observations of Changes in the Neuronal Biomarker "NAA" in many Human Brain Disorders. Bioenerg

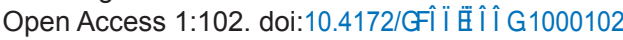

to maintain normal neuronal activity. This requirement is supported by evidence from human inborn errors in the tri-cellular metabolism of NAA and NAAG which result in profound structural and cognitive impairments attesting to the essential nature of this metabolic sequence in brain function [15]. This operating system is found everywhere in the brain and always requires four cell types for its completion. Since these four cell types represent the minimum required for the physiological operation of the NAA-NAAG system, their association has been described as the hierarchal "structural unit" of the brain, with each unit consisting of one neuron and at least one each of the other three cell types including oligodendrocytes, astrocytes and vascular endothelial cells [1]. Based on a nominal value of 25,000 neurons/ $\mathrm{mm}^{3}$ in human brain [35], there could be this number of single-neuron containing structural units $/ \mathrm{mm}^{3}$, with each unit exhibiting its own NAA-NAAG tri-cellular metabolic sequence, and operating within a four cell structural domain. A proposed structural unit of brain is illustrated in Figure 1.

Neuron signaling defines the "functional unit" of the brain: The function of neurons is to transmit information about their surrounding universe that can be used in eliciting behaviors appropriate for survival, and at least two neurons are required for this semiotic purpose. Thus, it was reasoned that a "functional unit" of the brain must be composed of the association of at least two single-neuron NAA-NAAG metabolicallydefined structural units [1]. These structural units in the vertebrate brain are connected to one another by a variety of mechanisms.
Between structural units, neurons are generally connected to each other at synapses (Syn), and astrocytes are connected to one another and to oligodendrocytes via gap junctions $(G j)$ that permit passage of relatively large molecules between cells. The endothelial cells are connected to each other by tight junctions $(T j)$ that restrict the free movement of all solutes, forming the capillary channels of the vascular system, and lastly, the ECF between cells forms contiguous channels, constituting a common highway for neurotransmitters, gliotransmitters, and traffic of all other imported and exported substances. Based on the estimated number of structural units per $\mathrm{mm}^{3}$, there could be 12,500 two-neuron functional units per $\mathrm{mm}^{3}$ of brain, with each unit exhibiting all of the basic signaling properties present in a complex brain.

While a single neuron may form thousands of synapses with other neurons, each forming a unique dendritic-synaptic-dendritic (DSD) structured field between them [36], every synapse that is formed is formed between just two neurons, and therefore these two neurons exist as a binary functional unit. Furthermore, while the number of synapses that form between two neurons is usually one [37], there is a "proximity factor" so that there may be as many as eight or more synapses formed between two neurons within a functional unit, with the number of synapses formed varying inversely with the distance of the neurons from one another [38,39]. It has also been proposed that it is the total number of dendritic connections and their characteristics that define the nature of each unique DSD field formed between any two neurons, that in turn interacts with neuronal frequency-encoded

A SINGLE NEURON

(NAA and NAAG synthases)

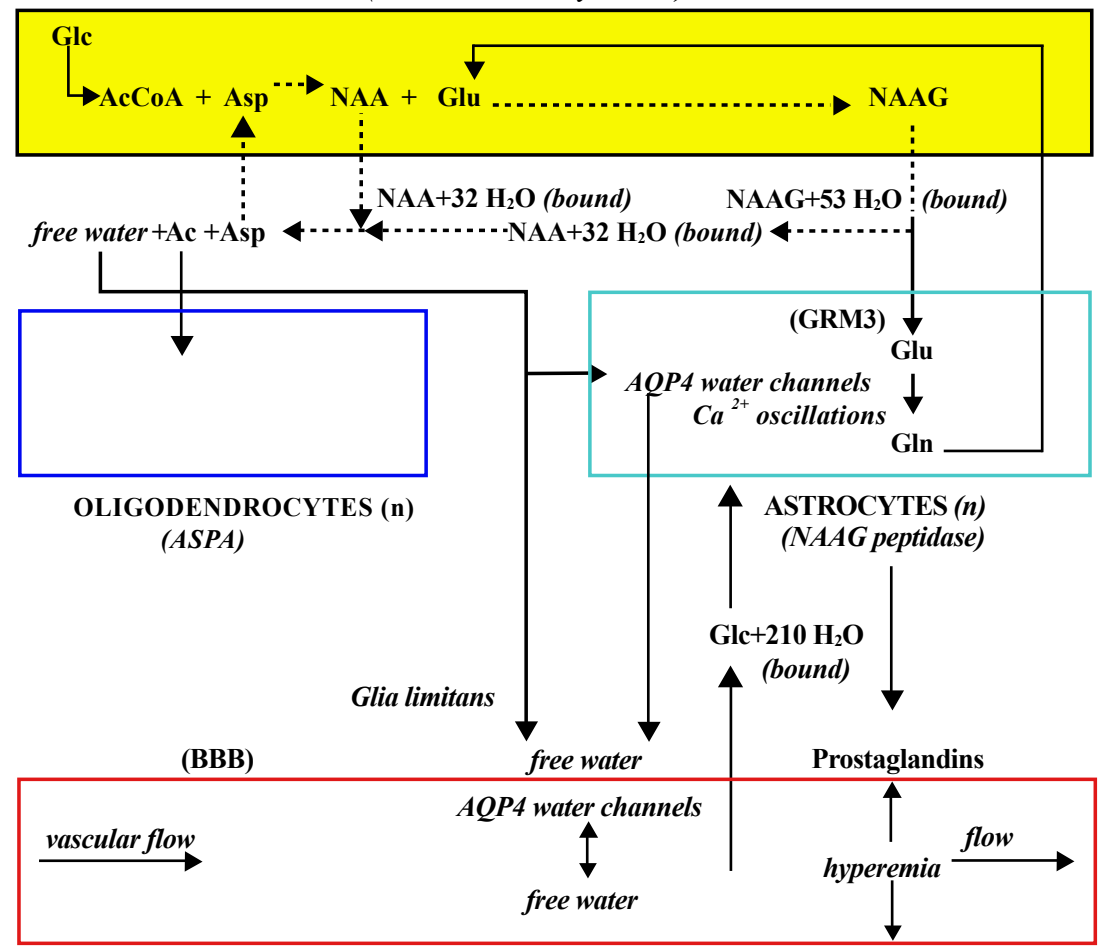

VASCULAR SYSTEM ENDOTHELIAL CALLS (N)

*Based on metabolic, physical and physiological properties of NAA and NAAG, this system serves neurons by (A) exporting metabolic water, (B) increasing focal sink capacity for metabolic waste products as well as heat, and $(C)$ obtaining sufficient energy supplies from the vascular system regardless of their momentary state of activation.

Figure 1: A proposed structural unit of the brain, a single neuron plus variable numbers of other cells in a four-cell bioenergetic operating system domain defined by the tri-cellular metabolism of NAA and NAAG* (adapted from [1]). 
Citation: Baslow MH, Burlina AP (2012) N-Acetylaspartate Metabolism Underlays the Structural and Functional Units of the Vertebrate Brain: A Bioenergetic Rationale for Clinical Observations of Changes in the Neuronal Biomarker "NAA" in many Human Brain Disorders. Bioenerg

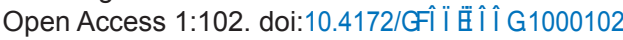

languages, memory formation and memory retrieval [36]. In cases where a single neuron forms synapses with many neurons, many binary functional units are formed, with each of their malleable DSD fields capable of learning and storing learned information as a memory. In Figure 2, a binary functional unit of brain, constructed from two conjoined structural units with their interconnections, is illustrated.

In this cartoon of a functional unit, the neuron box represents all classifications of neurons, all neurotransmitter types, all transporters and receptors, all neuronal configurations, and all of their wired (synaptic) and volume (ECF) mechanisms of communication. The oligodendrocyte and astrocyte boxes, respectively, represent all of their cellular sub-types and interactions, including the formation of myelin sheaths by oligodendrocytes, and all of their transporters and receptors. The vascular endothelial cell box represents all ECF-capillary interactions at the glia limitans of the blood-brain barrier (BBB), and all endothelial cell receptors and transporters. Each cell type described is also deemed to contain the complete genetic component of the organism with the precise nature of each cell determined by the specific genes expressed in that cell type. In this figure, the functional unit as illustrated is a representation that only rarely can be observed directly as a discreet entity. This is because a functional unit is always formed as a result of complex cellular associations. In these associations, macroglial cells that form a binary functional unit are extensively morphed in order to establish their various intercellular connections, the surrounding ECF follows tortuous pathways between these cells, and neurons which establish their synaptic connectivity via axonal-dendritic extensions may be long distances from one another in three-dimensional brain space [34]. Because signal transmissions within functional units as well as between functional units are organized, targeted and meaningful [1], loss of a single functional unit, along with its unique DSD field, will be of some consequence to overall brain functionality.

\section{A bioenergetic rationale for using the NAA-NAAG metabolic system as a biomarker for neuronal abundance and/or viability}

If an NAA metabolism-based functional unit as illustrated exists, then one may ask why this conceptual model may have value as a tool for understanding complex brain functions and its pathology. In this regard, it is proposed that the hierarchal functional unit model offers several important advantages in such investigations. This is based on two premises; one, that all normal brain functions are initiated at the level of binary functional units, and two, that all brain disorders are manifest at the level of functional units. Here, the bioenergetic importance of the underlying NAA-NAAG metabolic system present in each functional unit becomes apparent, in that measurement of its components can indicate brain functional conditions that exist at focal, regional and global brain levels at any given moment.

Neurons function by virtue of their connectivity, a product of ongoing synaptogenesis. Thus, logic dictates that all complex and rapid brain functions depend on underlying synaptic neuronal activity which in turn must be initiated at the level of a single functional unit, two neurons that communicate intelligibly with one another. Since every synapse that is formed in the brain is formed between two neurons at the level of a single functional unit, every message generated and transmitted within the brain must start with a single afferent neuron in a functional unit, and every thought process and philosophy resulting from this initial message then depends on the proper functioning of many additional serial and parallel synaptically interconnected functional units. It follows that every focal, regional and global brain pathology involving neurons, macroglia, ECF or the vascular system will also be evident at the level of a functional unit. Thus, using the two-neuron functional unit as a model, almost all brain genetic,

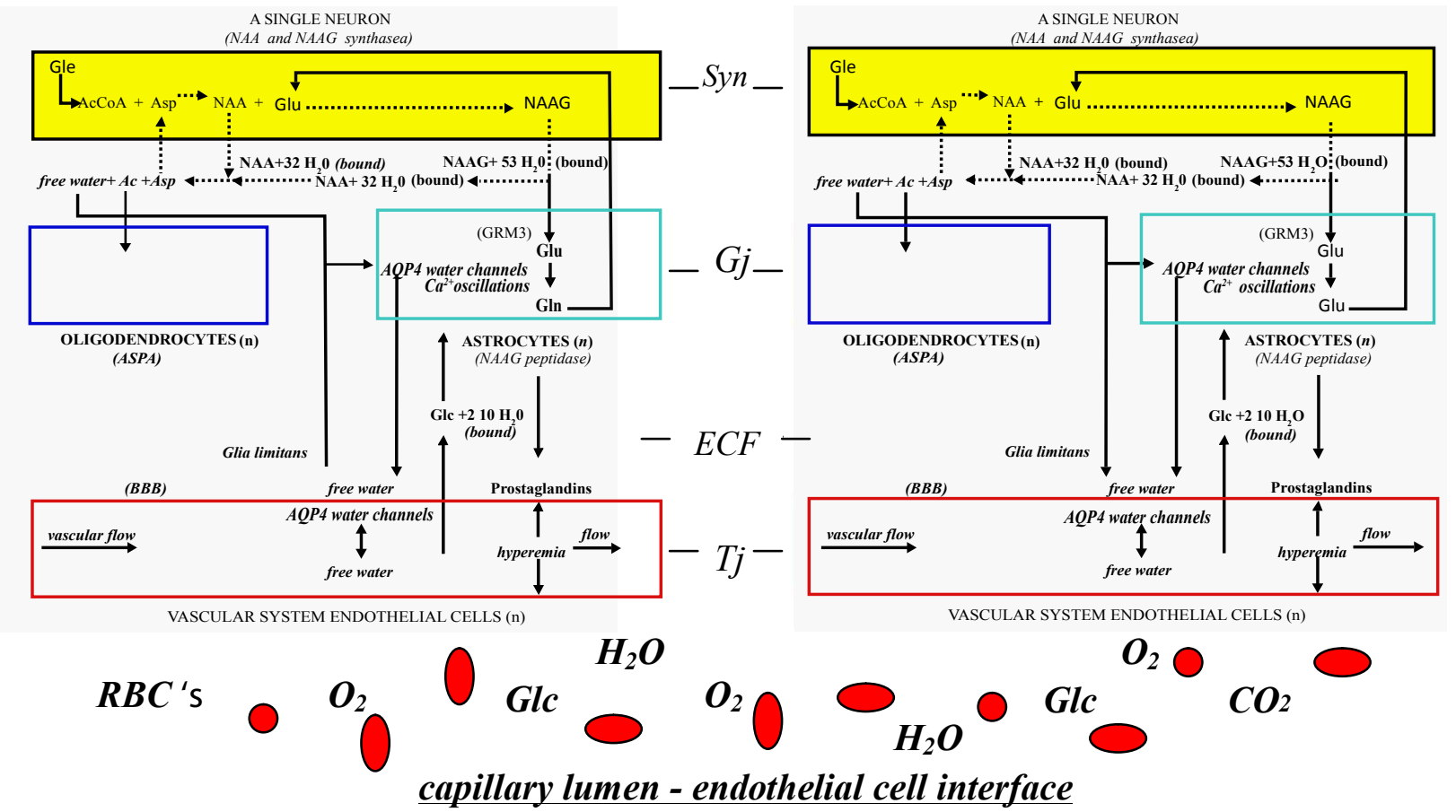

Figure 2: A proposed neuron-defined functional unit of the brain, two interconnected structural units, each containing a three-cell metabolic unit and a four-cell physiological operating system, here shown at a capillary lumen - endothelial cell interface (adapted from [1]). 
Citation: Baslow MH, Burlina AP (2012) N-Acetylaspartate Metabolism Underlays the Structural and Functional Units of the Vertebrate Brain: A Bioenergetic Rationale for Clinical Observations of Changes in the Neuronal Biomarker "NAA" in many Human Brain Disorders. Bioenerg

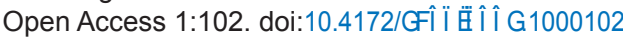

pathogen and degeneration-induced neuron pathologies, as well as results of brain traumas of all kinds, and many brain cell transporter, receptor and enzymatic drug interaction sites can be identified and located using this two-neuron cellular metabolic-physiological model as a map. In this way, it may be possible to envision and identify the sites of many brain diseases at a much simpler cellular level in terms of their specific genetic defects, failed cellular interactions, impaired neuronal connectivity, and disturbed relationships between the cellular and extra-cellular compartments. In addition, if the functional unit is the generator of rapid frequency-encoded signaling activity, and brain disorders are evident at the level of functional units, then results of any treatment protocols targeting metabolites, enzymes, receptors, genes or their products, or other components that effectively correct such disorders will take place at this level as well. Therefore, it is proposed that the functional unit concept can not only serve as a useful model for understanding and interpreting specific brain pathologies on a cellular level, but may also be a useful tool in developing drug and other treatment protocols for such disorders and predicting global outcomes of such treatments. This may be in terms of anticipated global improvements in specific brain functions based on metabolic, physiological or genetic alterations effected at the functional unit level.

\section{Use of NAA and NAAG as biomarkers in assessing brain condition}

Changes in NAA, NAAG, and total NAA and NAAG (tNAA) can reflect normal or abnormal brain functions: tNAA is not a static entity although its level has been recorded to be relatively stable in humans for periods of years, yet exhibiting a fairly large coefficient of variation during such long periods [40]. However, there is evidence that tNAA levels can change over minutes, hours, days or even months, and that these changes reflect their normal role in supporting demands of moment to moment changes in neuronal activities. In the following sections, interactions that occur between tNAA and physiological activities in normal brain as well as in several human brain disorders are discussed. How these changes are reflected at the level of proposed structural and functional units are also discussed. In each case, the role of the NAA-NAAG metabolic-physiological neuron communication support system is evaluated with regard to the nature and course of each of these interactions. In addition to many other available biomarkers [41], the metabolites NAA and NAAG are widely used as specific neuronal biomarkers in assessing global brain conditions based on minimally invasive methodologies afforded by magnetic resonance spectroscopy (MRS) and magnetic resonance spectroscopic imaging (MRSI). However, in most assessments, signals from NAA and NAAG are not separated and have been reported either as NAA, "NAA" or tNAA, although most of the MR signal observed is due to that of NAA since it is more abundant and produces a stronger MRS signal than does NAAG.

NAA and NAAG and their ${ }^{1} \mathrm{H}-\mathrm{NMR}$ spectroscopy: Applications of in vivo MR spectroscopy utilize nuclei such as ${ }^{13} \mathrm{C},{ }^{19} \mathrm{~F} .{ }^{31} \mathrm{P}$ and ${ }^{23} \mathrm{Na}$. However proton $\left({ }^{1} \mathrm{H}\right)$ spectroscopy provides the highest sensitivity and therefore is more widely used in studies of neurological disorders [31]. It is also fortuitous that in addition to NAA being present in high abundance in neurons, the acetyl methyl group of NAA produces a very strong proton MR resonance signal at $2.02 \mathrm{ppm}$. NAAG which is present in whole brain at about $10 \%$ of the level of NAA, produces a much weaker singlet resonance signal at $2.04 \mathrm{ppm}$, and cannot easily be separated from the NAA signal at most magnetic strengths and methodologies used in human studies. However, a method for determination of NAAG in human brain using an absolute reference method and linear combination of model spectra (LC Model) has been developed [42] and applied [43], although this technique cannot always be used to reliably separate NAA from NAAG [44].

Recently, it has been reported that NAA and NAAG could be measured separately in humans at a high magnetic strength, 7 Tesla ( $\mathrm{T})$, using difference editing based on the $\mathrm{CH} 2$ proton resonances of the aspartyl groups of NAA and NAAG [45]. In another investigation it has been reported that using MRS at an echo time of $140 \mathrm{~ms}$ and with a Gaussian broadening technique, that NAA and NAAG could also be reliably measured separately at $3 \mathrm{~T}$ [11]. Based on the difference editing technique in the human frontal brain [45], NAAG in gray and white matter was reported to be 13 and $28 \%$ of the concentration of NAA respectively, supporting results of previous chemical analyses that indicated that NAAG concentrations were higher in white matter than in gray matter and therefore constituted a larger fraction of tNAA in white matter [46]. Postmortem chemical analyses of normal human brains verify the MRS finding that NAAG is approximately $13 \%$ of NAA in gray matter and also indicate that NAA/NAAG ratios in specific gray matter brain regions can vary, with NAAG ranging from $10-18 \%$ of the concentration of NAA in these regions [24].

In most clinical settings, only magnetic strengths of 1.5 and $3 \mathrm{~T}$ with lower resolving power are commonly available for MRS diagnostic applications. Based on the ability to easily measure tNAA using this technique, even without a full understanding of either NAA and NAAG functions, but with the reasonable assumption that they serve as specific markers for neuron abundance and/or viability, tNAA has become the one of the most measured index substances in brain, and is widely used both as an indicator of normal brain function and as a diagnostic tool in most brain pathologies. In some studies, NAA has been reported as an absolute value based on use of the LC Model or by using brain phantoms containing NAA and/or other metabolites. However, in most cases, tNAA data is provided as a relative value, sometimes using brain water as an internal standard, or by using ratios of tNAA with other internal brain metabolites. While such ratios can and do provide reasonable clinical evidence and are useful in disease diagnosis in individual cases, it is also understood that such ratios involve two unknowns, either or both of which may change. In some cases involving fMRI studies where dynamic changes in tNAA in response to specific brain activities such as visual stimulation were tested, the initial resting state tNAA signal has been used as a control.

Since tNAA is a composite entity, the ratio between NAA and NAAG may also vary in ways specific to particular brain disorders. For example, in a postmortem study of schizophrenia patients' brains using chemical analyses, it was observed that whereas NAA was significantly lower in different brain regions, NAAG levels were normal [24]. In a related in vivo study of schizophrenia, it was also observed that NAA and NAAG varied independently in different brain regions, and in addition, that changes in their relative concentrations were significantly associated with different disease symptoms [11]. An important factor to be considered when using NAA and NAAG as biomarkers of neuronal integrity is that they are present almost exclusively in neurons in the CNS, whereas metabolites to which tNAA is often compared are more widely distributed in cells, so that a change in a given tNAA/metabolite ratio may not be indicative a loss of neurons or their viability, but of changes in abundance or viability in other cellular compartments.

tNAA can vary with brain size and age: NAA and NAAG content appear to change with brain age in mice, with older brains generally having significantly lower concentrations of these substances. At mouse postnatal day $3(0.42 \mathrm{wks})$ the tNAA value in whole brain was $43 \mu \mathrm{mol} / \mathrm{g}$ 
Citation: Baslow MH, Burlina AP (2012) N-Acetylaspartate Metabolism Underlays the Structural and Functional Units of the Vertebrate Brain: A Bioenergetic Rationale for Clinical Observations of Changes in the Neuronal Biomarker "NAA" in many Human Brain Disorders. Bioenerg Open Access 1:102. doi:10.4172/2167-7662.1000102

protein; at 3 wks, 90 , and in adults at 16-32 wks, it was 24 [47]. In this study it was also reported that the \% protein in brain remained constant between 3-32 weeks eliminating this as a reason for the apparent decline in tNAA. Brain shrinkage with age may also be a factor in assessing changes in tNAA levels. In a human longitudinal study of whole brain tNAA in mature individuals 25-35 years of age it was noted that over a period of 4 years, that while the average whole brain tNAA of $12.1 \mathrm{mM}$ did not change significantly, that there was a small significant reduction in brain parenchyma volume during this period [48] suggesting that there may have been some loss of functional units with age. In another study based on absolute measures of NAA and choline, it was observed that there was a significant decrease in the NAA/choline ratio with age of between $5-8 \%$ per decade [49]. Thus, age, associated growth stage, and brain size, especially where there may be significant differences due to gender, are important factors to be considered when using NAA, NAAG or tNAA as neuronal markers for assessing brain disorders.

The reason for ongoing changes during early developmental stages is probably associated with the changing numerical relationships between neuronal and other cells as the brain increases in size [35]. During these growth stages of the brain after most neurons are formed, neurons become associated in binary functional units primarily by producing axonal elongations and more complex dendritic arbors, whereas chaperone cells which do not contain NAA that are in functional units appear to increase in number by recruitment from precursor cells in order to accommodate these neuronal axonal extensions. This suggests that in cases of age related brain shrinkage, similar numerical changes between neuron and other cell densities are to be expected and that these will be reflected in altered tNAA measures.

Changes in NAA and NAAG reflect normal physiological variability: Changes in NAA and NAAG may also be due to normal reversible physiological variability within the NAA-NAAG system that can occur over minutes, hours, days or months. As examples, in the normal human visual cortex, after 10 min of visual stimulation, the tNAA signal was found to be reduced by about $7-10 \%$ [50,51], and in a study of sleep deprivation, it was observed that after $40 \mathrm{~h}$ without sleep, that the "NAA/water" ratio in the visual cortex was significantly decreased, also by approximately 7\% [52]. Environmental stress-related physiological variability in NAA metabolism over even longer periods may play a role in post-traumatic stress disorder and other neurological insults where tNAA is observed to be reduced, as well as in cases of environmental enrichment where tNAA levels rise. Examples of dynamic relationships between tNAA and human physiology have been observed, and in a course of treatment for chronic pain using slowfrequency trans-cranial magnetic stimulation, it was reported that a significant reduction in pain lasting several weeks was correlated with an increase in the tNAA signal [53]. In a human study of changes in hippocampal NAA in response to 4 months of a daily spatial navigation task, it was found that "NAA" increased by up to $2.5 \mathrm{mM}(+25 \%)$, and then returned to baseline within 4 months following termination of training [10].

Changes in NAA and NAAG may reflect drug interactions: There have been many studies where changes in tNAA have been observed in response to drug administration. In these cases, transient changes in tNAA have been found to reflect long or short term drug interactions that occur over periods of minutes to days. As examples, tNAA has been reported to be significantly reduced in humans and mice by intake of ethanol $[54,55]$ and to be increased over a period of days in mice by acamprosate ( $\mathrm{N}$-acetylhomotaurine) and related substances [55].

\section{Inborn errors in "NAA" metabolism affect bioenergetic mechanisms at the level of structural units}

There are two human inborn errors in the metabolism of NAA and NAAG that result in profound cognitive and motor function impairments. One is Canavan disease (hyperacetylaspartia), an osmotic disease where the absence of ASPA activity in oligodendrocytes is associated with the buildup of NAA and NAAG in brain ECF. The other is the lack of NAA synthase activity in neurons (hypoacetylaspartia) which results in the absence of both NAA and NAAG in neurons. The biochemical, metabolic and physiological consequences of these inborn errors appear to be directly associated with failure of the brains' NAA "operating system" at the level of a "structural unit".

Canavan disease (hyperacetylaspartia): Canavan disease (CD) is a rare globally distributed early-onset spongiform leukodystrophy [5658]. It is genetic in nature, and the syndrome results from a number of different mutations in the gene that encodes for ASPA, all of which result in inactive forms of this enzyme instead of the normal enzyme that is expressed in oligodendrocytes. The $\mathrm{CD}$ mutations are autosomally inherited and recessive traits, and usually, but not always $[59,60]$ result in development of the CD phenotype and in early mortality. CD is a progressive disease, and clinical symptoms associated with the typical $\mathrm{CD}$ phenotype are observed within the first few months of life. These early symptoms are characterized by initial apathy and difficulty in holding the head erect, as well as in an abnormally large head size. As the disease progresses, there is usually optic atrophy, a pronounced megaloencephaly, ataxia, myoclonus and some form of epilepsy. At later stages there is considerable psychomotor and mental retardation and a failure to meet all normal developmental milestones such as holding the head up, grasping at objects, speech and walking. As the disease progresses, spasticity becomes evident. In $\mathrm{CD}$, the most important clinical clue to disease diagnosis, and one that distinguishes it from all other leukodystrophies, is the finding of a $20-30 \%$ increase in levels of tNAA in the brain and the presence of elevated NAA and NAAG in cerebrospinal fluid (CSF), blood and urine of these patients [61]. Pathologically, CD is an "encephalitis periaxialis diffusa" in which the myelinating oligodendrocytes in white matter fail, and in so doing leave axons intact but unsheathed and therefore without normal function [34]. The spongiform condition is due to the presence of large vacuoles that form mostly, but not exclusively, in white matter where they are observed primarily in the intra-myelinic spaces of disrupted myelin sheaths. CD is considered to be an osmotic disease characterized by osmotic lesions in the form of elevated levels of NAA-water and NAAGwater in ECF compartments.

Hypoacetylaspartia: A second inborn error in NAA metabolism has been found in a single human case of lack of brain NAA and NAAG [62]. While the early effects in this case were reported to be comparatively mild, and with a slight delay in myelination, a follow-up of this patient revealed that by age 8 , the effects were profound, with microcephaly, marked abnormalities in motor performance, language ability and cognition, and with episodic status epilepticus [63]. This patient at about 13 years of age provided DNA that was used in a study to find the nature of the defect responsible for the disease syndrome. Genetic analysis of the DNA in this singular case revealed that the patient was homozygous for a 19 base-pair deletion in the genetic code for production of $\mathrm{N}$-acetyltransferase $8 \mathrm{~L}$, the enzyme that synthesizes NAA from AcCoA and Asp [2], and that this appears to be the primary deficiency associated with this disease. As a result of this defect, NAA is not synthesized, and therefore NAAG is also not able to be synthesized. The outcome of this inborn error is that the normal physiological 
Citation: Baslow MH, Burlina AP (2012) N-Acetylaspartate Metabolism Underlays the Structural and Functional Units of the Vertebrate Brain: A Bioenergetic Rationale for Clinical Observations of Changes in the Neuronal Biomarker "NAA" in many Human Brain Disorders. Bioenerg Open Access 1:102. doi:10.4172/2167-7662.1000102

Page 7 of 10

functions of NAA and NAAG in brain are absent, strongly supporting the notion that normal NAA and NAAG metabolism is essential for proper brain function. This unique case is also important in that it demonstrates for the first time that NAA and NAAG are not required for neuronal signaling in vivo, nor for interaction with oligodendrocytes in order to form myelin sheaths.

Changes in NAA, NAAG or tNAA associated with global brain disorders at the level of functional units

While many brain disorders are reflected in global changes in brain function that are associated with observable altered behaviors and/or metabolic imbalances, the basis for these diseases are initiated at the cellular level within the hierarchal functional units of the brain. The minimally invasive MR technology has allowed its wide use in measuring the normal levels and regional distribution of various brain metabolites, as well as for studies of the areas of brain activation under stimulatory conditions. Importantly, it has allowed the exploration of changes in any of these components that might be specifically associated with a variety of neurological and psychiatric disorders.

Based on evidence that NAA and NAAG are specific neuronal markers, and on the belief that these substances generally reflect neuron abundance and/or viability, they have been measured in almost all cases involving clinically observed psychological, toxicological and neurological pathologies. In most cases, significant correlations, sometimes transient, have been found between these clinical syndromes and brain tNAA levels in isolated brain areas, or in regional or global brain tNAA levels, as well as in ratios of tNAA with other important brain metabolites as observed in amyotrophic lateral sclerosis (ALS) [64]. These changes may be brought about by impaired neuron activity within functional units without extensive loss of neurons as in $\mathrm{CD}$ where tNAA is elevated, and in hypoacetylaspartia where tNAA is absent, or by reductions in tNAA due to loss of neurons as occurs in Alzheimer's disease (AD) [40]. Since NAA and NAAG are present in abundance in axons as well as soma, changes in tNAA may also reflect quantitative differences in synaptic and/or dendritic connectivity between functional units without either neuron loss or altered neuron viability. Perhaps this is the case in certain psychiatric disorders such as schizophrenia where tNAA is reduced but without clear evidence of any cellular pathology [65].

\section{Interpretation of clinical NAA, NAAG or tNAA results}

While tNAA measures in a wide variety of brain disorders are commonly made, interpretation of results remains problematic. However, in all of these cases, based on evidence of the physiological importance of the NAA-NAAG system in brain, and its proposed bioenergetic role in providing activated neurons with increased energy supplies, it can be anticipated that as a result of any positive or negative differences in measured NAA, NAAG or tNAA levels from established norms, including in their ratios with other metabolites or water, there will also be some measurable impact on cognitive functioning at the level of the global neuronal network. Thus, while various measures of tNAA when compared to population norms can provide a reasonable estimate of the condition and/or abundance of neurons, understanding the precise nature of the meaning of such changes related to brain function is still very difficult. To summarize, observed changes in regional brain NAA, NAAG or tNAA may be due many different factors including but not limited to:

\section{Metabolic failures in the NAA system}

${ }^{*}$ Specific metabolic failures in components of the NAA-NAAG metabolic system as in $\mathrm{CD}$ and in hypoacetylaspartia.
${ }^{*}$ Non-specific inborn errors in components of the NAA-NAAG system that affect its operation such as changes Asp/Glu transport mechanisms, or loss of AQP4 water channels

\section{Drug interactions}

${ }^{*}$ Specific drug interactions with the NAA tri-cellular metabolic system such as by the use of NAAG peptidase inhibitors.

${ }^{*}$ Non-specific drug interactions such as resulting from ethanol or lithium intake.

\section{Changes in neuron physiology}

${ }^{*}$ Natural or induced reversible long or short-term events affecting neuron signaling and physiology including acute focused stimulation and longer-term learning.

\section{Changes in neuron viability}

${ }^{\star}$ Reversible changes in short or long-term neuron viability such as may occur in cases of transient global or regional ischemic episodes, pathogen invasions, or to some forms of traumatic brain injuries.

${ }^{*}$ Irreversible changes in neuron viability as in some cases $\mathrm{CO}$ poisoning or long-term coma.

\section{Changes in neuron density}

*Permanent neuron loss as in AD.

\section{Changes in neuron connectivty}

${ }^{*}$ Changes in normal inter-neuronal connectivity without neuron loss as in schizophrenia.

\section{Changes in neuron ratios to other cells}

*Altered ratios of neuron volume to volumes of other cellular and extra-cellular brain compartments in a given region as occurs in cases of ECF or cellular edema, CSF expansion, astrogliosis, or certain malignancies.

\section{Changes in distant neuronal connections}

${ }^{*}$ Axonal pathologies or discontinuities far removed from a given brain region of interest as in concussion, spinal cord injuries, multiple sclerosis or ALS.

\section{Differences due to brain size, sex and age}

${ }^{\star}$ Normal differences associated with growth stage, sex and age.

${ }^{*}$ Abnormal regional or global differences in these factors due to various pathologies.

Indeed, changes in the neuron biomarker tNAA may be due to any and all combinations of these factors, and these differences are compounded further when tNAA is compared to different metabolites and/or water that are not only present in neurons, but are also present in other types of cells and other brain compartments. A downside of using tNAA as a biomarker for human brain diseases is that it is non-specific and many unrelated conditions may show reduced tNAA levels. Clearly, use of such tNAA observations in clinical diagnoses and for evaluation of potential outcomes, while a valuable assessment tool, will benefit substantially from additional information concerning other biomarkers specific to each pathology [66]. A generalized picture of how various brain traumas and diseases may interact at the level of structural and functional units in the brain is presented in figure 3. Shown in this 
Citation: Baslow MH, Burlina AP (2012) N-Acetylaspartate Metabolism Underlays the Structural and Functional Units of the Vertebrate Brain: A Bioenergetic Rationale for Clinical Observations of Changes in the Neuronal Biomarker "NAA" in many Human Brain Disorders. Bioenerg Open Access 1:102. doi:10.4172/2167-7662.1000102

Page 8 of 10

figure is a single functional unit of the many that exist in synaptically linked chains of serial and parallel interconnected units. Myriad of such units, functioning in concert, are considered to comprise the brains neuronal network. While redundancies exist, failure of any unit for any reason is of some consequence to the network. In this cartoon, a binary functional unit is depicted as being at the epicenter of many global brain disorders.

\section{Conclusions}

In this article we have presented information regarding what is currently known about the metabolism and physiology of NAA and NAAG in the vertebrate brain. From this information, several concepts have emerged. First, that the NAA metabolic system, while not required for individual neuronal survival, signaling or myelination, is nonetheless essential for normal cognitive brain function, and therefore that clinical use of measures of any of its biochemical and enzymatic components as biomarkers of normal brain function is reasonable. Second, that the NAA-NAAG system appears to be the basic operating system of the brain and that this operating system constitutes an important homeostatic bioenergetic mechanism for maintaining the osmotic integrity of the brain and for supplying adequate energy to activated neurons in all regions of the brain regardless of the level of neuronal activity.

The unique tri-cellular metabolic nature of the NAA system requiring enzymes that are differentially distributed in neurons, oligodendrocytes and astrocytes has also led to the hypothesis that the smallest structural unit of the brain that can exist must include at least one each of these cells, and that their physiological roles require an additional cell, an endothelial cell that in concert with other endothelial cells form the network of capillaries of the brain vascular system. Together, these four cell types are considered to constitute the minimal structural unit of the brain. Moreover, since the brains function is communication, a process requiring at least two neurons, the minimal functional unit of the brain must be made up of at least two single neuron structural units, and that a complex brain is composed of many of these binary functional units operating in an extensive neuronal network.

Since the NAA-NAAG metabolic-physiological bioenergetic system underlies the normal operation of neurons in individual functional units throughout the brains neuronal network, it is also clear that measurements of these substances as biomarkers in various brain regions, albeit with the caveats listed, can provide reasonable indications of both normal and abnormal brain function. In addition,

\section{Canavan disease, hypoacetylaspartia and other neurogenetic metabolic diseases that affect the brains "operating system"}

\section{Alzheimer's disease, and other neuronal cell-loss pathologies and dementias that result in inactive functional units}

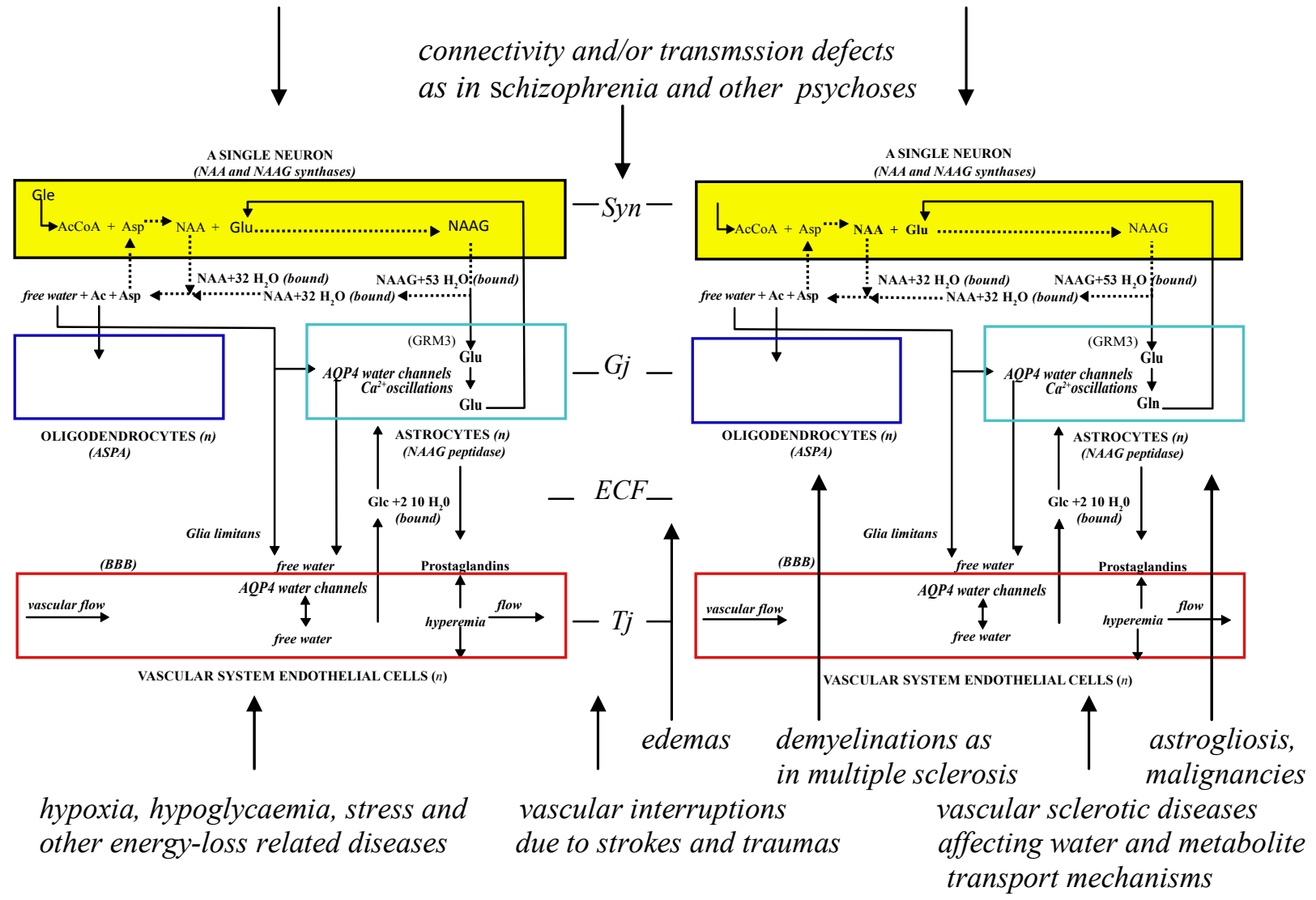

Figure 3: Global brain diseases and traumas that can be traced to malfunctions at the level of the NAA-NAAG defined "structural unit", and/or the neuron-defined binary "functional unit". 
Citation: Baslow MH, Burlina AP (2012) N-Acetylaspartate Metabolism Underlays the Structural and Functional Units of the Vertebrate Brain: A Bioenergetic Rationale for Clinical Observations of Changes in the Neuronal Biomarker "NAA" in many Human Brain Disorders. Bioenerg Open Access 1:102. doi:10.4172/2167-7662.1000102

such measurements may sometimes serve as useful clinical indicators in evaluating possible outcomes associated with specific conditions such as in cases of stroke. If the functional unit hypothesis is correct, then in many cases global brain disorders can be traced to specific bioenergetic failures that occur within the operation of functional units. While loss or malfunction of a single functional unit may diminish brain capacity to some extent, loss of functionality of multiple units, evident as changes in the biomarkers NAA, NAAG or tNAA, would be expected to reflect various degrees of failure, the pathological expressions of which focally, regionally or globally may be associated with a wide variety of different human brain disorders. As a corollary, changes that occur spontaneously, or from various therapies and interventions that result in normalization of these biomarkers would be expected to reflect recovery from such disorders.

\section{References}

1. Baslow MH (2011) Biosemiosis and the cellular basis of mind. How the oxidation of glucose by individual neurons in brain results in meaningful communications and in the emergence of "mind". Biosemiotics 4: 39-53.

2. Wiame E, Tyteca D, Pierrot N, Collard F, Amyere M, et al. (2010) Molecular identification of aspartate $\mathrm{N}$-acetyltransferase and its mutation in hypoacetylaspartia. Biochem J 425: 127-136.

3. Becker I, Lodder J, Gieselmann V, Eckhardt M (2010) Molecular characterization of N- acetylaspartylglutamate synthetase. J Biol Chem 285: 29156-29164.

4. Collard F, Stroobant V, Lamosa P, Kapanda CN, Lambert DM, et al. (2010) Molecular identification of $\mathrm{N}$ - acetylaspartylglutamate synthase and $\beta$-citrylglutamate synthase. J Biol Chem 285: 29826-29833.

5. Baslow MH (2000) Functions of $\mathrm{N}$-acetyl-L-aspartate and $\mathrm{N}$-acetyl-Laspartylglutamate in the vertebrate brain. Role in glial cell-specific signaling. $J$ Neurochem 75: 453-459.

6. Baslow MH, Suckow RF, Sapirstein V, Hungund BL (1999) Expression of aspartoacylase activity in cultured rat macroglial cells is limited to oligodendrocytes. J Mol Neurosci 13: 47-53.

7. Bitto E, Bingman CA, Wesenberg GE, McCoy JG, Phillips GN Jr (2007) Structure of aspartoacylase, the brain enzyme impaired in Canavan disease. Proc Natl Acad Sci USA 104: 456-461.

8. Mesters JR, Barinka C, Li W, Tsukamoto T, Majer P, et al. (2006) Structure of glutamate carboxypeptidase II, a drug target in neuronal damage and prostate cancer. EMBO J 25: 1375-1384.

9. Sacha P, Zamecnik J, Barinka C, Hlouchová K, Vícha A, et al. (2007) Expression of glutamate carboxyeptidase II in human brain. Neuroscience 144: 1361-1372.

10. Lövden M, Schaefer S, Noack H, Kanowski M, Kaufmann J, et al. (2011) Performance-related increases in hippocampal $\mathrm{N}$-acetylaspartate (NAA) induced by spatial navigation training are restricted to BDNF Val homozygotes. Cereb Cortex 21: 1435-1442.

11. Jessen F, Fingerhut N, Sprinkart AM, Kuhn KU, Petrovsky N, et al. (2011) $\mathrm{N}$-acetylaspartylglutamate (NAAG) and $\mathrm{N}$-acetylaspartate (NAA) in patients with schizophrenia. Schizophren Bull.

12. Birken DL, Oldendorf WH (1989) N-acetyl-L-aspartic acid: a literature review of a compound prominent in $1 \mathrm{H}-\mathrm{NMR}$ spectroscopic studies of brain. Neurosci Biobehav Rev 13: 23-31.

13. Baslow MH (1997) A review of phylogenetic and metabolic relationships between the acylamino acids, $\mathrm{N}$-acetyl-L-aspartic acid and $\mathrm{N}$-acetyl-L-histidine, in the vertebrate nervous system. J Neurochem 68: 1335-1344.

14. Baslow MH (2007) N-Acetylaspartate and N-Acetylaspartylglutamate. In: Lajtha A Handbook of Neurochemistry and Molecular Neurobiology. ( $3^{\text {rd }}$ Edition), Springer Science, NY.

15. Baslow MH (2010) Evidence that the tri-cellular metabolism of $\mathrm{N}$-acetylaspartate functions as the brain's "operating system": how NAA metabolism supports meaningful intercellular frequency-encoded communications. Amino Acids 39: $1139-1145$

16. Baslow MH, Guilfoyle DN (2002) Effect of N-acetylaspartic acid on the diffusion coefficient of water: A proton magnetic resonance phantom method for measurement of osmolyte-obligated water. Anal Biochem 311: 133-138.

17. Mehta SK, Kaur K, Sharma S, Bhasin KK (2007) Behavior of acetyl modified amino acids in reverse micelles: A non-invasive and physiochemical approach. J Colloid Interface Sci 314: 689-698.

18. Plumridge TH, Waigh RD (2002) Water structure theory and some implications for drug design. J Pharm Pharmacol 54: 1155-1179.

19. Baslow MH (1999) The existence of molecular water pumps in the nervous system: a review of the evidence. Neurochem Int 34: 77-90.

20. Baslow MH (2002) Evidence supporting a role for $\mathrm{N}$-acetyl-L-aspartate as a molecular water pump in myelinated neurons in the central nervous system. An analytical review. Neurochem Int 40: 295-300.

21. Agre P, King LS, Yasui M, Guggino WB, Ottersen OP, et al. (2002) Aquaporin water channels-from atomic structure to clinical medicine. J Physiol 542: 3-16.

22. Buffoli B (2010) Aquaporin biology and nervous system. Curr Neuropharmaco 8: $97-104$

23. Andrew RD, Labron MW, Boehnke SE, Carnduff L, Kirov SA (2007) Physiological evidence that pyramidal neurons lack functional water channels. Cereb Cortex 17: 787-802.

24. Reynolds LM, Reynolds GP (2011) Differential regional N-acetylaspartate deficits in postmortem brain in schizophrenia, bipolar disorder and major depressive disorder. J Psychiatr Res 45: 54-59.

25. Bammer R, Stollberger R, Augustin M, Simbrunner J, Offenbacher $\mathrm{H}$, et al (1999) Diffusion-weighted imaging with navigated interleaved echo-planar imaging and a conventional gradient system. Radiology 211: 799-806.

26. Baslow MH (2008) The astrocyte surface NAAG receptor and NAAG peptidase signaling complex as a therapeutic target. Drug News Perspect 21: 251-257.

27. Chopra M, Yao Y, Blake TJ, Hampson DR, Johnson EC (2009) The neuroactive peptide $\mathrm{N}$-acetylaspartylglutamate is not an agonist at the metabotropic glutamate receptor subtype 3 of metabotropic glutamate receptor. J Pharmacol Exp Ther 330: 212-219.

28. Fricker AC, Mok MH, de la Flor R, Shah AJ, Woolley M, et al. (2009) Effects of $\mathrm{N}$-acetylaspartylglutamate (NAAAG) at group II mGluRs and NMDAR. Neuropharmacology 56: 1060-1067.

29. Zonta M, Angulo MC, Gobbo S, Rosengarten B, Hossmann KA, et al. (2003) Neuron-to-astrocyte signaling is central to the dynamic control of brain microcirculation. Nat Neurosci 6: 43-50.

30. ladecola C, Nedergaard M (2007) Glial regulation of the cerebra microvasculature. Nat Neurosci 10: 1369-1376.

31. Baslow MH, Guilfoyle DN (2007) Using proton magnetic resonance imaging and spectroscopy to understand brain "activation". Brain Lang 102: 153-164.

32. Xu HL, Mao L, Ye S, Paisansathan C, Vetri F, et al. (2008) Astrocytes are a key conduit for upstream signaling of vasodilation during cerebral cortical neuronal activation in vivo. Am J Physiol Heart Circ Physiol 294: H622-H632.

33. Attwell D, Buchan AM, Charpak S, Lauritzen M, Macvicar BA et al. (2010) Glia and neuronal control of brain blood flow. Nature 468: 232-243.

34. Nave KA (2010) Myelination and support of axonal integrity by glia. Nature 468: 244-252.

35. Herculano-Houzel S (2011) Not all brains are made the same: new views on brain scaling in evolution. Brain Behav Evol 78: 22-36.

36. Baslow MH (2009) The languages of neurons; An analysis of coding mechanisms by which neurons communicate, learn and store information. Entropy 11: 782-797

37. Nauen DW (2011) Methods of measuring activity at individual synapses: A review of techniques and the findings they have made possible. J Neurosci Methods 194: 195-205.

38. Kalisman N, Silverberg G, Markram H (2003) Deriving physical connectivity from neuronal morphology. Biol Cybern 88: 210-218.

39. Sepanyants A, Hirsch JA, Martinez LM, Kisvarday ZF, Ferecsko AS, et al. (2008) Local potential connectivity in cat primary visual cortex. Cereb Cortex 18: $13-28$. 
Citation: Baslow MH, Burlina AP (2012) N-Acetylaspartate Metabolism Underlays the Structural and Functional Units of the Vertebrate Brain: A Bioenergetic Rationale for Clinical Observations of Changes in the Neuronal Biomarker "NAA" in many Human Brain Disorders. Bioenerg Open Access 1:102. doi:10.4172/2167-7662.1000102

40. Kirov II, George IC, Jayawickrama N, Babb JS, Perry NN, et al. (2012) Longitudinal inter- and intra-individual human brain metabolic quantification over 3 years with proton MR spectroscopy at 3T. Magn Reson Med 67: 27-33.

41. Ward M, Schofield EL (2010) Biomarkers for brain disorders. Therapy 7: 321 336.

42. Provencher SW (1993) Estimation of metabolite concentrations from localized in vivo proton NMR spectra. Magn Reson Med 30: 672-679.

43. Hájek M, Burian M, Dezortová M (2000) Application of LC Model for quality control and quantitative in vivo $1 \mathrm{H}$ MR spectroscopy by short echo time STEAM sequence. MAGMA 10: 6-17.

44. Wellard RM, Briellmann RS, Jennings C, Jackson GD (2005) Physiologic variability of single-voxel proton MR spectroscopic measurements at 3 T. AJNR Am J Neuroradiol 26: 585-590.

45. Choi C, Ghose S, Uh J, Patel A, Dimitrov IE, et al. (2010) Measurement of $\mathrm{N}$-acetylaspartylglutamate in the human frontal brain by $1 \mathrm{H}-\mathrm{MRS}$ at $7 \mathrm{~T}$. Magn Reson Med 64: 1247-1251.

46. Pouwels PJ, Frahm J (1997) Differential distribution of NAA and NAAG in human brain as determined by quantitative localized proton MRS. NMR Biomed 10: 73-78.

47. Yao FS, Caserta MT, Wyrwicz AM (1999) In vitro proton and phosphorus NMR spectroscopic analysis of murine (C57BI/6J) brain development. NMR Biomed 12: $463-470$

48. Rigotti DJ, Kirov II, Djavadi B, Perry N, Babb JS, et al. (2011) Longitudinal whole-brain $\mathrm{N}$-acetylaspartate concentration in healthy adults. AJNR Am J Neuroradiol 32: 1011-1015.

49. Gruber S, Bogner W, Stadlbauer A, Krssak M, Bodamer O (2011) Magnetic resonance spectroscopy in patients with Fabry and Gaucher disease. Eur J Radiol 79: 295-298.

50. Sarchielli P, Tarducci R, Presciutti O, Gobbi G, Pelliccioli GP, et al. (2005) Functional $1 \mathrm{H}-\mathrm{MRS}$ findings in migraine patients with and without aura assessed interictally. Neuroimage 24: 1025-1031.

51. Baslow MH, Hrabe J, Guilfoyle DN (2007) Dynamic relationship between neurostimulation and $\mathrm{N}$-acetylaspartate metabolism in the human visual cortex: evidence that NAA functions as a molecular water pump during visual stimulation. J Mol Neurosci 32: 235-245.

52. Urrila AS, Hakkarainen A, Heikkinen S, Huhdankoski O, Kuusi T, et al. (2006) Preliminary findings of proton magnetic spectroscopy in occipital cortex during sleep deprivation. Psychiatry Res 147: 41-46.

53. Fregni F, Potvin K, Dasilva D, Wang X, Lenkinski RE, et al. (2011) Clinica effects and brain metabolic correlates in non-invasive cortical neuromodulation for visceral pain. Eur J Pain 15: 53-60.
54. Gomez R, Behar KL, Watzl J, Weinzimer SA, Gulanski B, et al. (2011) Intravenous ethanol infusion decreases human cortical gamma-aminobutyric acid and $\mathrm{N}$-acetylaspartate as measured with proton magnetic resonance spectroscopy at 4 Tesla. Biol Psychiatry.

55. Baslow MH, Suckow RF, Hungund BL (2000) Effects of ethanol and of alcoho dehydrogenase inhibitors on the reduction of $\mathrm{N}$-acetylaspartate levels of brain in mice in vivo: a search for substances that may have therapeutic value in the treatment of Canavan disease. J Inherit Metab Dis 23: 684-692.

56. Matalon R, Michals K, Sebesta D, Deanching M, Gashkoff P, et al. (1988) Aspartoacylase deficiency and $\mathrm{N}$-acetylaspartic aciduria in patients with Canavan disease. Am J Med Genet 29: 463-471.

57. Baslow MH, Resnik TR (1997) Canavan disease: Analysis of the nature of the metabolic lesions responsible for development of the observed clinical symptoms. J Mol Neurosci 9: 109-125.

58. Assadi M, Janson C, Wang DJ, Goldfarb O, Suri N, et al. (2010) Lithium citrate reduces excessive intra-cerebral $\mathrm{N}$-acetylaspartate in Canavan disease. Eur $\mathrm{J}$ Paediatr Neurol 14: 354-359.

59. Janson CG, Kolodny EH, Zeng BJ, Raghavan S, Pastores G, et al. (2006) Mild onset presentation of Canavan's disease associated with novel G212A point mutation in aspartoacylase gene. Ann Neurol 59: 428-431.

60. Velinov M, Zellers N, Styles J, Wisniewski K (2008) Homozygosity for mutation G212A of the gene for aspartoacylase is associated with atypical form of Canavan's disease. Clin Genet 73: 288-289.

61. Burlina AP, Ferrari V, Divry P, Gradowska W, Jakobs C, et al. (1999) $\mathrm{N}$-acetylaspartylglutamate in Canavan disease: an adverse effector? Eur J Pediatr 158: 406-409.

62. Martin E, Capone A, Schneider J, Hennig J, Thiel T (2001) Absence of $\mathrm{N}$-acetylaspartate in the human brain: Impact on neurospectroscopy? Ann Neurol 49: 518-521.

63. Boltshauser E, Schmitt B, Wevers RA, Engelke U, Burlina AB, et al. (2004) Follow-up of a child with hypoacetylaspartia. Neuropediatrics 35: 255-258.

64. Carew JD, Nair G, Andersen PM, Wuu J, Gronka S, et al. (2011) Presymptomatic spinal cord neurommetabolic findings in SODI-positive people at risk for familia ALS. Neurology 77: 1370-1375.

65. Brugger S, Davis JM, Leucht S, Stone JM (2011) Proton magnetic resonance spectroscopy and illness stage in schizophrenia- a systematic review and meta-analysis. Biol Psychiatry 69: 495-503.

66. Steen RG, Hamer RM, Lieberman JA (2005) Measurement of brain metabolites by $1 \mathrm{H}$ magnetic resonance spectroscopy in patients with schizophrenia: A systematic review and meta-analysis. Neuropsychopharmacol 30: 1949-1962. 